levels. In iSLE patients, IFN scores and BAFF levels were significantly increased compared to HCs. Also, IFN scores correlated with proportions of switched memory B-cells, plasma cells, IgG levels and correlated negatively with complement levels in iSLE patients.

Conclusions In this cross-sectional study, distortions in B-cell subsets were observed in iSLE patients and were correlated with IFN scores and IgG levels. Since these factors play an important role in the pathogenesis of SLE, iSLE patients with these distortions, high IFN scores, and high levels of IgG and BAFF may be at risk for progression to SLE.

Acknowledgement This study was funded by ReumaNederland

\section{P101 HYDROXYCHLOROQUINE SUPPRESSES IFN-INDUCIBLE GENES AND BAFF IN PATIENTS WITH INCOMPLETE AND NEW ONSET SYSTEMIC LUPUS ERYTHEMATOSUS}

Wietske Lambers, Johanna Westra, Hendrika Bootsma, Karina de Leeuw. Dept. of Rheumatology and Clinical Immunology, University of Groningen, University Medical Centre Groningen, Groningen, The Netherlands

\subsection{6/lupus-2020-eurolupus. 145}

Background Hydroxychloroquine (HCQ) is the backbone of treatment in Systemic Lupus Erythematosus (SLE). It has been suggested that this drug can also delay the onset of SLE in patients with lupus symptoms, who do not meet the classification criteria yet. Interferon (IFN) type $\mathrm{I}$ is an early mediator in the pathogenesis of SLE. IFN-gamma induced protein 10 (IP-10) and B-cell activating factor (BAFF) are increased in SLE, but also prior to SLE diagnosis and correspond with disease activity. The purpose of this study is to analyze the effects of HCQ on IFN-induced gene expression, serum IP-10 and BAFF levels.

Methods Patients with incomplete SLE (ANA titer $\geq 1: 80$, symptoms $<5$ years, $\geq 1$ objectified clinical ACR criterion), or new onset SLE (based on ACR 1997 or SLICC criteria) were included if there was a clinical indication to start HCQ treatment. Blood samples were taken at the start and after 16 weeks. Apart from NSAIDs, no other immunosuppressive drugs were initiated. The IFN-inducible genes MX1, IFI44L and LY6E were measured in whole blood by RT-PCR. An IFN score was determined by the normalized $\log$ (relative expression) of these 3 genes. Serum levels of IP-10 and BAFF were measured using ELISA. Differences between the time points were statistically assessed with Wilcoxon test.

Results In total, nine patients were included: six with iSLE and three with new onset SLE. The median SLEDAI was 4. After 16 weeks of treatment with HCQ, the relative expression of all three IFN-inducible genes decreased in 8 of 9 patients, and the IFN score decreased significantly $(p=0.012)$. There was a trend towards lower IP-10 levels $(p=0.078)$, and a significant decrease in BAFF-levels $(\mathrm{p}=0.023)$ after treatment with HCQ. The median SLEDAI decreased to 0 . The one incomplete SLE patient who had no decrease in IFN score, developed SLE. (See figure 1.)

Conclusion After start of treatment with HCQ in patients with incomplete or new onset SLE, IFN score and BAFF levels decreased significantly, and there was a trend towards lower IP-10 levels. As these are early mediators in SLE, this might indicate that HCQ could delay disease progression in incomplete and new onset SLE. However, larger sample size and longer follow up is needed.

Acknowledgement This study was funded by ReumaNederland
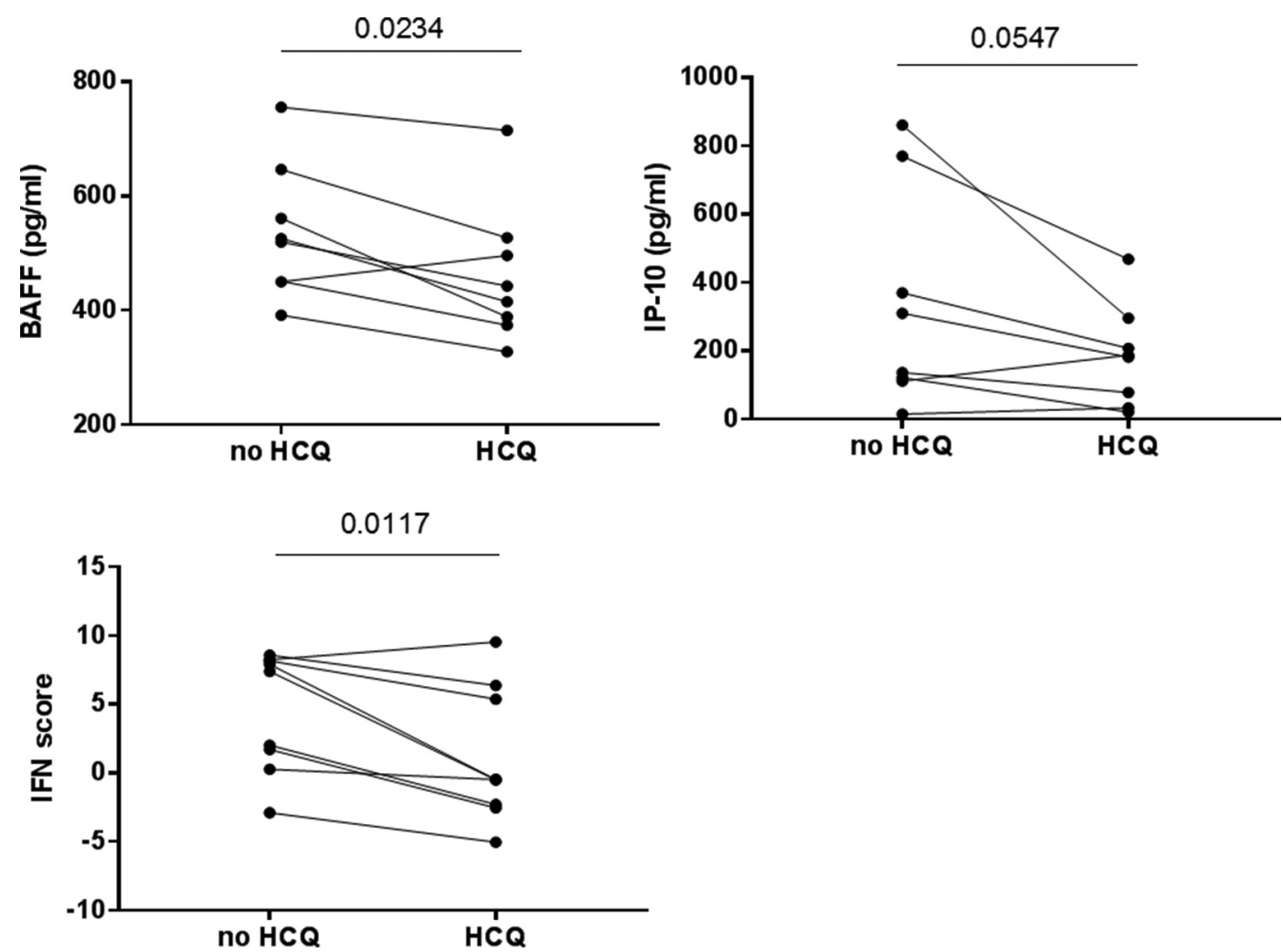

Abstract P101 Figure 1 Plasma levels of B-cell activating factor, Interferon-gamma induced protein 10, and calculated IFN score before and 16 weeks after starting hydroxychloroquine. P-values are shown 